\title{
ON DISCONJUGATE DIFFERENTIAL SYSTEMS
}

\author{
PHILIP HARTMAN and AUREL WINTNER
}

1. Introduction. Let $F, G$ (and all capital letters to be used below) denote $n$ by $n$ matrices the elements of which are real-valued continuous functions on an interval $a \leqslant t \leqslant b$. Correspondingly, by a solution $x=x(t)$ of a differential system

$$
\left(G(t) x^{\prime}\right)^{\prime}+F(t) x=0,
$$

with $\operatorname{det} G(t) \neq 0$, or of a differential system

$$
x^{\prime \prime}+G(t) x^{\prime}+F(t) x=0,
$$

will be meant a (vector) solution all $n$ components of which are real-valued.

The trivial solution $x(t) \equiv 0$ will not be referred to as a solution. Following the manner of speaking introduced in $(7)$ in the scalar case $(n=1)$, let (1) or (2) be called disconjugate on the interval $a \leqslant t \leqslant b$ if there does not exist a solution $x(t)$ satisfying

$$
x\left(t_{1}\right)=0 \text { and } x\left(t_{2}\right)=0 \text { if } a \leqslant t_{1}<t_{2} \leqslant b .
$$

The following considerations deal with various facts concerning disconjugate vector equations, (1) or (2), for an arbitrary $n$. Sections $3-5$ concern an extension of a result (2, p. 66) from self-adjoint systems (1) to non-self-adjoint systems (1). Sections 6-9 deal with criteria which are sufficient in order that the system (2) be disconjugate; these criteria can be considered analogues of the trivial condition $F \leqslant 0$ in the case that (2) is a scalar equation. Section 10 concerns convexity properties of solutions of (2). In $\$ 11$, these properties are used to obtain an extension of the result of (6) on the half-line $0 \leqslant t<\infty$.

2. Notations. Since every matrix $A=\left(a_{i k}\right)$ is supposed to be real, its transposed matrix $\left(a_{k i}\right)$ will be denoted by $A^{*}$. Correspondingly, the symmetric part of $A$ is its Hermitian part, that is, the matrix $\frac{1}{2}\left(A+A^{*}\right)$. The latter will be denoted by $A^{\circ}$. Thus $A=A^{\circ}$ if and only if $A$ is symmetric $\left(=A^{*}\right)$.

By $A \leqslant B$ or $B \geqslant A$ will be meant that the quadratic form belonging to $(B-A)^{\circ}=B^{\circ}-A^{\circ}$ is non-negative definite (that is, that $(B-A)^{\circ} \geqslant 0$, where 0 denotes the zero matrix). Accordingly, $A \leqslant B$ is equivalent to $A^{\circ} \leqslant B^{\circ}$. Similarly, $A<B$ or $B>A$ is defined to mean that (the quadratic form belonging to the symmetric matrix) $B^{\circ}-A^{\circ}$ is positive definite (that is, $A^{\circ}<B^{\circ}$ or $B^{\circ}>A^{\circ}$ ).

3. On a theorem of Morse. In terms of the definition given in connection with (3), the simplest case of a theorem of Morse (6, p. 66), a theorem which

Received February 18, 1955. 
extends Sturm's comparison theorem from the scalar case to the case of vectors, can be formulated as follows:

(I) (Morse) Suppose that the pair of coefficient matrices $P_{j}, Q_{j}$ of the linear differential systems

$$
\left(P_{j}(t) x^{\prime}\right)^{\prime}+Q_{j}(t) x=0,
$$

where $j=1,2$, satisfy the following conditions at every t on $a \leqslant t \leqslant b$ :

$$
0<P_{1} \leqslant P_{2}, \quad Q_{1} \geqslant Q_{2},
$$

and

$\left(6_{j}\right)$

$$
P_{j}=P_{j}^{*}, \quad Q_{j}=Q_{j}^{*} .
$$

Then $\left(4_{2}\right)$ is disconjugate on $a \leqslant t \leqslant b$ whenever $\left(4_{1}\right)$ is disconjugate there.

Here the real matrices $P_{j}, Q_{j}$ (and later on $F, G, \ldots$ ) are supposed to be given as continuous functions on $a \leqslant t \leqslant b$.

It will turn out that the above-quoted consequence of Morse's comparison theorem can be refined so as to eliminate for $\left(4_{2}\right)$, but not for $\left(4_{1}\right)$, the restriction to be self-adjoint, that is, the restriction $\left(6_{2}\right)$ :

(II) The assertion of (I) remains true if, instead of (5), $\left(6_{1}\right)$ and $\left(6_{2}\right)$, only (5) and $\left(6_{1}\right)$ are assumed.

It follows from the refinement (II) of (I) that, without assuming (1) to be self-adjoint, the disconjugate character of (1) can be assured by exhibiting for (1) a scalar Sturmian majorant. In fact, it is clear that (II) contains the following

Corollary. Let $p(t)$ and $q(t)$ be scalar continuous functions satisfying

$$
0<p(t) I \leqslant G(t) \text { and } q(t) I \geqslant F(t),
$$

where $I$ is the unit matrix and $a \leqslant t \leqslant b$. Then (1) must be disconjugate on $a \leqslant t \leqslant b$ whenever the scalar equation

$$
\left(p(t) x^{\prime}\right)^{\prime}+q(t) x=0
$$

is disconjugate there.

Note that (7) implies that $G(t)>0$. Conversely, if $G(t)>0$, then (7) can be satisfied by choosing $p(t)$ to be the least eigenvalue of $G^{\circ}(t)$, and $q(t)$ the greatest eigenvalue of $F^{\circ}(t)$, for every fixed $t$.

4. Reduction to the self-adjoint case. Clearly, the extension (II) of (I) can be concluded from (I) and from the following

Lемма 1. If the differential system

$$
\left(G^{\circ}(t) x^{\prime}\right)^{\prime}+F^{\circ}(t) x=0,
$$

where $G^{\circ}(t)>0$, is disconjugate on $a \leqslant t \leqslant b$, then (1) is disconjugate there. 
It should be noted that the assumption $\operatorname{det} G \neq 0$ in (1) is implied by the assumption $G^{\circ}>0$ in (9); in fact, it is known (cf. 3) that $\operatorname{det} G \geqslant \operatorname{det} G^{\circ}$ whenever $G^{\circ}>0$.

The proof of Lemma 1 will depend on an adaptation of Morse's proof of (I). Before proving Lemma 1 , it will be shown that its converse is false: If (1) is disconjugate on $a \leqslant t \leqslant b$, then (9) need not be, not even when $G^{\circ}>0$ (hence $\operatorname{det} G^{\circ}>0$ ). This is shown by the following example:

Let $n=2$ and $a=0, b=\pi$, let $G$ (hence $G^{\circ}$ ) be the unit matrix and let

$$
F=\left(\begin{array}{ll}
0 & 0 \\
2 & 0
\end{array}\right) \text {, hence } F^{\circ}=\left(\begin{array}{ll}
0 & 1 \\
1 & 0
\end{array}\right) \text {, and } x=\left(\begin{array}{l}
u \\
v
\end{array}\right) \text {. }
$$

Then (1) and (9) become

$$
u^{\prime \prime}=0, v^{\prime \prime}+2 u=0 \text { and } u^{\prime \prime}+v=0, v^{\prime \prime}+u=0,
$$

respectively. But the general solution of the first of these two systems is

$$
u=c_{1} t+c_{2}, \quad v=-\frac{1}{3} c_{1} t^{3}-c_{2} t^{2}+c_{3} t+c_{4}
$$

which implies that if both $u$ and $v$ vanish at some $t=t_{1}$ and another $t=t_{2}$, then $u$ and $v$, hence the vector $x$, must vanish for all $t$. In other words, the present case of (1) is disconjugate on every interval $a \leqslant t \leqslant b$. On the other hand, the present case of $(9)$ fails to be disconjugate on $0 \leqslant t \leqslant \pi$, since $(u, v)=(\sin t, \sin t)$ is a solution satisfying $x(0)=0=x(\pi)$.

5. Proof of Lemma 1. Suppose that the assertion of Lemma 1 is false. Then the assumptions of Lemma 1 are satisfied but (1) is not disconjugate on $a \leqslant t \leqslant b$; so that (1) has a solution $x(t) \equiv 0$ satisfying (3) for some $t_{1}, t_{2}$. Since the definition of disconjugate behavior on an interval is such as to imply disconjugate behavior on any subinterval of that interval, it can be assumed that $t_{1}=a$ and $t_{2}=b$, hence

$$
x(a)=0, \quad x(b)=0,
$$

but $x(t) \not \neq 0$. Scalar multiplication of $(1)$ by $x(t)$, when followed by integration over $a \leqslant t \leqslant b$ and a partial integration of the result, leads to

$$
\int_{a}^{b}\left(x^{\prime} \cdot G x^{\prime}-x \cdot F x\right) d t=0
$$

by (10). This can be written in the form

$$
\int_{a}^{b}\left(x^{\prime} \cdot G^{\circ} x^{\prime}-x \cdot F^{\circ} x\right) d t=0
$$

since $z \cdot A z=z \cdot A^{\circ} z$, by the definition of $A^{\circ}$ in $\$ 2$.

On the other hand, since (9) is supposed to be disconjugate on $a \leqslant t \leqslant b$, it is clear that $\lambda_{0}>0$, if $\lambda_{0}$ denotes the least eigenvalue of the boundary value problem (10) of (9). But $\lambda_{0}>0$ means that 


$$
\min \int_{a}^{b}\left(x^{\prime} \cdot G^{\circ} x^{\prime}-x \cdot F^{\circ} x\right) d t>0,
$$

where the min refers, for instance, to the class of all continuously differentiable vector functions $x(t), a \leqslant t \leqslant b$, satisfying (10) and

$$
\int_{a}^{b} x \cdot x d t=1 .
$$

Since (12) and (13) contradict (11), the proof of Lemma 1 is complete.

6. A generalization of the Schwarz invariant. The following considerations will deal with (2), rather than (1). The results of the next section can be motivated by the remark that the matrix

$$
E=F-\frac{1}{2} G^{\prime}-\frac{1}{4} G^{2}
$$

reduces to Schwarz's invariant (cf. e.g., 5, p. 121) in the case that (2) is a scalar equation. The connection between (2) and (14) is given by the following

Lemma 2. Let $F(t)$ be continuous, and $G(t)$ continuously differentiable, on $a \leqslant t \leqslant b$. Let $R=R(t)$ be a solution of

$$
R^{\prime}=-\frac{1}{2} G(t) R \text { and } \operatorname{det} R \neq 0 .
$$

Then the substitution

$$
x=R(t) y
$$

transforms (2) into

$$
y^{\prime \prime}+B(t) y=0, \quad B=R^{-1} E R
$$

where $E=E(t)$ is given by $(14)$.

The existence of matrices $R(t)$ satisfying (15) is clear. In fact, such a matrix results by placing $R=\left(r_{1}, \ldots, r_{n}\right)$, where the column vectors $r_{k}=r_{k}(t)$ are $n$ linearly independent, and otherwise arbitrary, solution vectors of the linear system $r^{\prime}=-\frac{1}{2} G(t) r$.

Proof of Lemma 2. Since $G(t)$ is assumed to have a continuous derivative, it follows from (15) that $R(t)$ has a continuous second derivation given by

$$
R^{\prime \prime}=-\frac{1}{2} G^{\prime} R-\frac{1}{2} G R^{\prime}=-\frac{1}{2} G^{\prime} R+\left(\frac{1}{2} G\right)^{2} R .
$$

A formal substitution of (16) into (2) gives the equation

$$
R y^{\prime \prime}+\left(2 R^{\prime}+G R\right) y^{\prime}+\left(R^{\prime \prime}+G R^{\prime}+F R\right) y=0 .
$$

The coefficient of $y^{\prime}$ is 0 , by virtue of (15). Hence, (19) can be written as $y^{\prime \prime}+B y=0$, where

$$
B=R^{-1}\left(R^{\prime \prime} R^{-1}+G R^{\prime} R^{-1}+F\right) R .
$$

Since $R^{\prime} R^{-1}=-\frac{1}{2} G$, by (15), and $R^{\prime \prime} R^{-1}=-\frac{1}{2} G^{\prime}+\left(\frac{1}{2} G\right)^{2}$, by (18), it follows that the matrix (20) is $R^{-1} E R$. This gives (17), hence Lemma 2 . 
Remark. In order to apply comparison theorems to (17), it is necessary to know bounds for the quadratic form $x . B x$. In general, a knowledge of such bounds depends on a knowledge of $R$. An exceptional case is the case of the following remark: If the coefficient $G(t)$ in (2) is skew-symmetric for every $t$, then $R(t)$ in (17) can be chosen to be a rotation matrix for every $t$; that is, $R^{*}=R^{-1}$ and $\operatorname{det} R=+1$, so that $B(t)$ is orthogonally equivalent to $E(t)$ for every fixed $t$. This can be seen as follows:

If $G^{*}=-G$, then it is readily verified from $(15)$ and $\left(R^{*}\right)^{\prime}=\left(R^{\prime}\right)^{*}$ that $R^{*} R^{\prime}+R^{* \prime} R=0$; so that $R^{*} R=$ const. for every solution $R=R(t)$ of (15). Hence, if the matrix $R(t)$ is determined by the initial condition $R(a)=I$, then $R^{*} R=$ const. $=I$, and the assertion follows.

7. Criteria for (2) to be disconjugate. By adapting to matrices a device used repeatedly by Picard (cf., e.g., 4, p. 8), the following criteria will now be proved:

(III) In order that (2) be disconjugate on $a \leqslant t \leqslant b$, it is sufficient that, for $a \leqslant t \leqslant b$, either

$$
F \leqslant-\frac{1}{4} G G^{*}
$$

or

$$
F \leqslant G^{\prime}-\frac{1}{4} G^{*} G
$$

where the existence of a continuous derivative $G^{\prime}$ is part of the assumption in the second case (but not in the first).

Note that, since $G(t)$ and $G^{*}(t)$ are not in general commutable, (22) does not reduce to (21) even if $G=$ const. (i.e., $G^{\prime}=0$ ).

Since $G G^{*}$ is a (symmetric) non-negative definite matrix for every $G$, condition (21) is relaxed if the $\frac{1}{4}$ is replaced by $\frac{1}{4}-\epsilon<\frac{1}{4}$. But it turns out that any such relaxation of $(21)$ is inadmissible: In (III), the constant $\frac{1}{4}$, occurring in (21) and (22), is the best possible constant. This can be seen as follows:

Let $n=2$ and

$$
G=\left(\begin{array}{rr}
0 & 1 \\
-1 & 0
\end{array}\right)
$$

finally $F=\lambda I$, where $\lambda$ is a constant. Then, since $G G^{*}=I=G^{*} G$ and $G^{\prime}=0$ in view of $(23)$, it is clear that the condition in which the $\frac{1}{4}$ of $(21)$ or $(22)$ is relaxed to $\frac{1}{4}-\epsilon$ is satisfied if $\lambda \leqslant-\frac{1}{4}+\epsilon$, for example, if

$$
F=\left(-\frac{1}{4}+\epsilon\right) I
$$$$
(\epsilon>0) \text {. }
$$

Hence it is sufficient to show that the case (23)-(24) of (2) fails to be disconjugate on the interval $a \leqslant t \leqslant b$, if the latter is suitably chosen (the matrices (23), (24) are independent of $t$ ).

First, since (23) implies that $G^{\prime}=0$ and $G^{2}=I$, it follows from (14) and (24) that $E=\epsilon I$. Hence the differential equation in (17) reduces to $y^{\prime \prime}+\epsilon y=0$ 
and admits therefore the (vector) solution $y(t)=I \sin \left(\epsilon^{\frac{1}{2}} t\right)$. Since the latter vector has more than one zero on $a \leqslant t \leqslant b$ if $\epsilon$ is fixed and $b-a$ is large enough, it follows from (16), where $\operatorname{det} R(t) \neq 0$, that (2) has a solution satisfying (10) if the interval $a \leqslant t \leqslant b$ is suitably chosen. This proves the last italicized statement.

Instead of proving (III) directly, the following generalization of (III) will be derived:

(IV) In order that (2) be disconjugate on $a \leqslant t \leqslant b$, it is sufficient that there exists a continuously differentiable matrix $K(t)$ satisfying

$$
F-K^{\prime} \leqslant-\left(\frac{1}{2} G-K^{\circ}\right)\left(\frac{1}{2} G^{*}-K^{\circ}\right)
$$

on $a \leqslant t \leqslant b$.

This criterion implies both assertions of (III). In fact, (25) reduces to (21) if $K=0$, and to $(22)$ if $K=G$.

8. Proof of (IV). Multiply (2) by $x$, substitute the value of the scalar product $x \cdot x^{\prime \prime}$ from the identity $x \cdot x^{\prime \prime}=\left(x \cdot x^{\prime}\right)^{\prime}-x^{\prime 2}$ and, under the assumption (3), integrate the result between $t_{1}$ and $t_{2}$. This gives the relation

$$
\int_{t_{1}}^{t_{2}}\left(x^{\prime 2}-x \cdot G x^{\prime}-x \cdot F x\right) d t=0 .
$$

If $K=K(t)$ is any matrix function possessing a continuous derivative, then, since

$$
(x \cdot K x)^{\prime}=x^{\prime} \cdot\left(K+K^{*}\right) x+x \cdot K^{\prime} x
$$

and $K+K^{*}=2 K^{\circ}$, and since

$$
\int_{t_{1}}^{t_{2}}(x \cdot K x)^{\prime} d t=0
$$

in view of $(3)$,

$$
\int_{t_{1}}^{t_{\mathbf{s}}}\left(2 x^{\prime} \cdot K^{\circ} x+x \cdot K^{\prime} x\right) d t=0 .
$$

If this identity is added to (25a) it follows that

$$
\int_{t_{1}}^{t_{2}}\left(\left|x^{\prime}-\left(\frac{1}{2} G^{*}-K^{\circ}\right) x\right|^{2}-x \cdot M x\right) d t=0,
$$

where $M=F-K^{\prime}+\left(\frac{1}{2} G-K^{\circ}\right)\left(\frac{1}{2} G^{*}-K^{\circ}\right)$.

Suppose that $M=M(t)$ satisfies the condition $M \leqslant 0$ throughout. By the definition of $M$, this is the case if $K=K(t)$ satisfies the inequality (25) for $a \leqslant t \leqslant b$. Then, since $M \leqslant 0$ means, by $\S 2$, that $y . M^{\circ} y \leqslant 0$ or, what is the same thing, that $y . M y \leqslant 0$ holds for every vector $y$, both terms of the integrand in $(26)$ are non-negative for $t_{1} \leqslant t \leqslant t_{2}$. It follows therefore from (26) that both of these terms, which are continuous functions, must vanish for all $t$ between $t_{1}$ and $t_{2}$. The vanishing of the first term for $t_{1} \leqslant t \leqslant t_{2}$ 
implies that the vector $x(t)$ is a solution of the differential equation $x^{\prime}=A(t) x$, where $A=\frac{1}{2} G^{*}-K^{\circ}$. Since this differential equation is of first order, and since its solution $x(t)$ satisfies, by (3), the initial condition $x\left(t_{1}\right)=0$, it follows from the uniqueness theorem of linear differential equations that $x(t) \equiv 0$.

This shows that if (25) is satisfied, then (3) implies $x(t) \equiv 0$ for every solution of (1). This proves (IV).

9. Another criterion. In what follows, an idea of Lichtenstein (1, p. 1298), applied by him to a partial differential equation of (elliptic type) for a scalar, will be adapted to (2), an ordinary differential equation for a vector $x$.

As in $\S 6$, suppose that $G(t)$ is continuously differentiable. Then (2) possesses the adjoint

$$
x^{\prime \prime}-G^{*}(t) x^{\prime}+\left(F^{*}(t)-G^{* \prime}(t)\right) x=0
$$

(in which the coefficient matrices are continuous functions). In fact, if $m(x)$ and $n(x)$ denote the (vector) differential operators on the left of (2) and (27), respectively, then, since $u . A v=v \cdot A^{*} u$, it is seen that

$$
y \cdot m(x)-x \cdot n(y)=y \cdot x^{\prime \prime}-x \cdot y^{\prime \prime}+y \cdot G x^{\prime}+y^{\prime} \cdot G x+y \cdot G^{\prime} x
$$

is an identity. Since the expression on the right of this identity is the derivative of $y \cdot x^{\prime}-x \cdot y^{\prime}+y \cdot G x$, the assertion follows.

We do not known an "elementary" verification of the following theorem (the analogue of which was used by Lichtenstein for his purposes (1, p. 1298)):

(V) Let $F(t)$ be continuous, and $G(t)$ continuously differentiable, on $a \leqslant t \leqslant b$. Then both (2) and (27) are disconjugate on $a \leqslant t \leqslant b$ if either (2) or (27) is disconjugate there.

The truth of this assertion follows directly from the definition of disconjugate behavior and from the fact that the pair of adjoint differential equations (2), (27) and the (common) boundary condition (3) determine Green kernels which are adjoint (i.e., transposed) kernels in the sense of Fredholm's theory.

Remark. If use is made of (V), then the second of the criteria in (III) follows from the first (and conversely). In fact, if the coefficients of (2) are replaced by those of $(27)$, then (21) goes over into

$$
F^{*}-G^{* \prime} \leqslant-\frac{1}{4}\left(-G^{*}\right)\left(-G^{*}\right)^{*}=-\frac{1}{4} G^{*} G
$$

a condition which can be written in the form (22), since $A \leqslant B$ is equivalent to $A^{*} \leqslant B^{*}(\S 2)$.

10. Convexity. The assumption (21) for the coefficients of (2) implies a geometrical restriction for the squared lengths of solution vectors of (2); cf. (6), where $G=0$.

LEMma 3. If $x(t)$ is any solution of (2), then the graph of $r(t)=|x(t)|^{2}$ in a $(t, r)$-plane is convex (from below) on every $t$-interval on which (21) is satisfied. 
This assertion is equivalent to the statement that the second derivative of $|x(t)|^{2}$ is non-negative by virtue of $(21)$. But if $K=0$, then (25) and

$$
r(t)=|x(t)|^{2}+\int^{t} x \cdot K x d t
$$

reduce to (21) and $|x(t)|^{2}$, respectively. Hence the assertion is contained in the statement that $(28)$ satisfies

$$
r^{\prime \prime} \geqslant 0
$$

by virtue of (25) and (2). But this can be verified as follows:

Let the expression (28), where $|x|^{2}=x . x$, be differentiated twice, and let $x^{\prime \prime}$ be substituted from (2) in the resulting representation of $r^{\prime \prime}$. It then follows that $\frac{1}{2} r^{\prime \prime}$ is the integrand in (26). Hence (29) follows from (25), the latter being equivalent to $x . M x \leqslant 0$. Thus Lemma 3 is verified.

11. Bounded and unbounded solutions. This lemma makes it possible to generalize the existence theorem of (6), where

$$
F(t) \leqslant 0, \quad G(t)=0,
$$

to the case of an arbitrary pair $F, G$ satisfying (21), as follows:

(VI) If the coefficients of (2) satisfy (21) for large positive t, then the system (2) of order $2 n$ possesses $n$ linearly independent solution vectors, say

$$
x_{1}(t), \ldots, x_{i}(t), \ldots, x_{n}(t),
$$

such that the lengths $\left|x_{i}(t)\right|$ of the vectors (31) tend to finite limits $(\geqslant 0)$ as $t \rightarrow \infty$, whereas $|x(t)| \rightarrow \infty$ holds for the lengths of all solutions vectors which are linearly independent of the $n$ particular solutions (31).

The proof of (VI) will not use (21) directly, but only its consequence, according to which

$$
r^{\prime \prime}(t) \geqslant 0, \text { where } r=|x|^{2},
$$

holds for all solutions $x=x(t)$ of $(2)$.

Proof of (VI). The first part of (III) assures that, if $t^{1}$ and $t^{2}\left(>t^{1}\right)$ are large enough, then (2) is disconjugate on $t^{1} \leqslant t \leqslant t^{2}$. Hence, for the same reasons as in (6, p. 363), there belongs to any pair of constant vectors $u, v$ a unique solution $x=x(t)$ of (1) satisfying

$$
x\left(t^{1}\right)=u, \quad x\left(t^{2}\right)=v .
$$

Without loss of generality, it can be assumed that $t^{1}=0$. Let $u$ be any unit vector (there are $\infty^{n}$ such choices of $u$ ) and, with reference to a fixed choice of $u$, let $x_{k}(t)$, where $k=1,2, \ldots$, denote the solution vector $x(t)$ belonging to the case $t^{1}=0, t^{2}=k, v=0$ of (33); so that

$$
\left|x_{k}(0)\right|=1, \quad x_{k}(k)=0 .
$$


It is clear from (34) and (32) that the sequence $x_{1}(t), \ldots, x_{k}(t), \ldots$ is uniformly bounded on every finite $t$-interval; in fact,

$$
\left|x_{k}(t)\right| \leqslant 1
$$$$
0 \leqslant t \leqslant k
$$

Hence, if the sequence of the derivatives is bounded at $t=0$, that is, if

$$
\left|x^{\prime}{ }_{k}(0)\right|<\text { const. as } k \rightarrow \infty,
$$

then the proof of (VI) can be completed in the same way as in (6, pp. 364-365). But (36) can be proved as follows:

Since $x^{\prime}{ }_{k}(t)$ is continuous, there exists on $0 \leqslant t \leqslant 1$ some $t^{k}$ satisfying

$$
\left|x^{\prime}{ }_{k}\left(t^{k}\right)\right|=\max _{0 \leqslant t \leqslant 1}\left|x_{k}^{\prime}(t)\right| \text {. }
$$

Let $\gamma$ denote a constant satisfying

$$
|F(t) x| \leqslant \gamma|x|, \quad|G(t) x| \leqslant \gamma|x| \quad(0 \leqslant t \leqslant 1)
$$

for every vector $x$. It can be supposed that $\gamma \geqslant 2$. Then, for every $k$, there is a number $\tau=\tau_{k}$ for which

$$
n \gamma|\tau|=1 \text { and } 0 \leqslant t^{k}+\tau \leqslant 1
$$

Only the algebraic sign of $\tau_{k}$ (and not $\left|\tau_{k}\right|$ ) depends on $k$. By the mean value theorem of differential calculus,

$$
x_{k}\left(t^{k}+\tau\right)=x_{k}\left(t^{k}\right)+\tau x_{k}{ }^{\prime}\left(t^{k}\right)+\frac{1}{2} \tau^{2} x_{k}{ }^{\prime \prime}(\theta),
$$

where $\theta$ is between $t^{k}$ and $t^{k}+\tau$. Actually, (40) is to be considered as a set of $n$ scalar equations, one for each component of $x_{k}$, and the value of $\theta$ varies from equation to equation but, in each case, is a number between $t^{k}$ and $t^{k}+\tau$.

It follows from (2) and (38) that the absolute value of the $j$ th component of $x_{k}{ }^{\prime \prime}(\theta)$ does not exceed $\gamma\left(\left|x_{k}{ }^{\prime}\left(\theta_{j}\right)\right|+\left|x_{k}\left(\theta_{j}\right)\right|\right)$, where $\theta=\theta_{j}$ is an intermediary value belonging to the $j$ th equation in (40). Since $\left|x\left(\theta_{j}\right)\right| \leqslant 1$, by (35), and $\left|x_{k}{ }^{\prime}\left(\theta_{j}\right)\right| \leqslant\left|x_{k}{ }^{\prime}\left(t^{k}\right)\right|$, by (37), it follows that $\left|x_{k}{ }^{\prime \prime}(\theta)\right| \leqslant n \gamma\left(\left|x_{k}{ }^{\prime}\left(t^{k}\right)\right|+1\right)$. In view of (35), neither $\left|x_{k}\left(t^{k}\right)\right|$ nor $\left|x_{k}\left(t^{k}+\tau\right)\right|$ exceeds 1 . Hence (40) implies that

$$
\left|\tau x_{k}{ }^{\prime}\left(t^{k}\right)\right| \leqslant 2+\frac{1}{2} n \tau^{2} \gamma\left(\left|x_{k}{ }^{\prime}\left(t^{k}\right)\right|+1\right)
$$

or

$$
|\tau|\left(1-\frac{1}{2} n|\tau| \gamma\right)\left|x_{k}{ }^{\prime}\left(t^{k}\right)\right| \leqslant 2+\frac{1}{2} n \tau^{2} \gamma
$$

From the first part of (39),

$$
\frac{1}{2}(n \gamma)^{-1}\left|x_{k}^{\prime}\left(t^{k}\right)\right| \leqslant 2+\frac{1}{2}(n \gamma)^{-1} .
$$

Consequently, the sequence $x_{1}{ }^{\prime}\left(t^{1}\right), x_{2}{ }^{\prime}\left(t^{2}\right), \ldots$ is bounded. In view of (37), this proves (36). 


\section{REFERENCES}

1. L. Lichtenstein, Article II C 12 (1924) in the Encyklopädie der Mathematischen Wissenschaften, II. $3_{2}$.

2. M. Morse, A generalization of the Sturm separation and comparison theorems in $n$-space, Math. Ann. 103 (1930), 52-69.

3. A. M. Ostrowski and O. Taussky, On the variation of the determinant of a positive definite matrix, Koninkl. Nederl. Akad. Wetensch., Proceedings (A), 54 (1951), 383-385.

4. E. Picard, Lȩ̧ons sur quelques problèmes aux limites de la théorie des équations différentièlles (Paris, 1930).

5. E. G. C. Poole, Introduction to the Theory of Linear Differential Equations (Oxford, 1936).

6. A. Wintner, On linear repulsive forces, Amer. J. Math., 71 (1949), 362-366.

7. - - On the non-existence of conjugate points, Amer. J. Math., 78 (1951), 368-380.

The Johns Hopkins University 